\title{
Liderança e Satisfação no Esporte Escolar: Teste da Hipótese da Congruência do Modelo Multidimensional de Liderança
}

\author{
Vinicius de Matos Rodrigues \\ Instituto Federal da Bahia, BA, Brasil.
}

\author{
Ana Alayde Werba Saldanha \\ Universidade Federal da Paraíba, PB, Brasil.
}

\begin{abstract}
Resumo: O Modelo Multidimensional de Liderança tem sido a abordagem teórica mais utilizada nos estudos de liderança no esporte. Segundo a sua principal proposição, conhecida como hipótese da congruência, quando os atletas percebem que o seu treinador apresenta o comportamento que eles preferem em um determinado contexto, o desempenho e a satisfação são maximizados. Contudo, os estudos que testaram a hipótese da congruência não confirmaram a sua validade. Além disso, a maior parte das investigações utilizou amostras de atletas adultos, fato que dificulta a transferência dos resultados para outros subgrupos populacionais. Nesse sentido, o presente estudo teve como objetivo testar a hipótese da congruência em uma amostra de atletas escolares. A amostra foi composta por 466 atletas com idade média de 16,16 (DP =0,79), que responderam um questionário sociodemográfico, a Escala de Liderança no Esporte (versões preferência e percepção) e o Questionário de Satisfação do Atleta (versão liderança). A análise da consistência interna e análise fatorial confirmaram a validade psicométrica dos instrumentos. Os resultados das análises de regressão suportam parcialmente a hipótese de que a satisfação dos atletas é dependente da congruência entre o comportamento do treinador percebido e preferido pelos atletas.
\end{abstract}

Palavras-chave: Liderança, Satisfação, Psicologia do Esporte, Avaliação Psicológica.

\section{Leadership and Satisfaction in Young Athletes: Testing the Hypothesis of Congruence of the Multidimensional Model of Leadership}

\begin{abstract}
The Multidimensional Model of Leadership has been the most widely used theoretical approach to studies of leadership in sport. According to its main proposition, known as the congruence hypothesis, when athletes perceive their coach shows the behavior they prefer on a given context, performance and satisfaction are maximized. However, studies that have tested the hypothesis of congruence did not confirm its validity. Furthermore, most of the research samples used adult athletes, a fact that complicates extrapolating the results to other subpopulations. In this sense, the present study aimed to test the hypothesis of congruence in a school athletes group. The sample consisted of 466 athletes, with an average age of 16.16 $(\mathrm{SD}=0.79)$, who completed: a demographic questionnaire; the Leadership Scale in Sport (preference and perception versions); and the Athlete Satisfaction Questionnaire (leadership version). Analysis of the internal consistency and factor analysis confirmed the psychometric validity of instruments. The results of the regression analysis partially support the hypothesis that athletes' satisfaction is dependent on the congruence between athletes' preferred and perceived coaches behavior.
\end{abstract}

Keywords: Leadership, Satisfaction, Sport Psychology, Psychological Assessment. 


\title{
El Liderazgo y la Satisfacción en el Deporte Escolar: Probando la Hipótesis de la Congruencia del Modelo Multidimensional de Liderazgo
}

\begin{abstract}
Resumen: El Modelo Multidimensional de Liderazgo ha sido el abordaje teórico más utilizado en los estudios de liderazgo en el deporte. Según su principal proposición, conocida como hipótesis de congruencia, cuando los deportistas perciben que su entrenador presenta un comportamiento que ellos prefieren en un determinado contexto, el desempeño y la satisfacción son maximizados. Todavía, los estudios que probaron la hipótesis de congruencia no confirman su validez. Además, la mayoría de las investigaciones utilizó muestras de deportistas adultos, hecho que dificulta la extrapolación de los resultados para otros subgrupos poblacionales. En este sentido, este estudio se propuso probar la hipótesis de congruencia en una muestra de deportistas escolares. La muestra fue compuesta por 466 atletas con edad media de 16, 16 (DT = 0,79), que contestaron: una encuesta socio demográfica, la Escala de Liderazgo en el Deporte (versión preferencia y percepción) y la Encuesta de Satisfacción del deportista (versión liderazgo). El análisis de consistencia interna y el análisis factorial confirmaron la validez psicométrica de los instrumentos. Los resultados de los análisis de regresión soportan parcialmente la hipótesis de que la satisfacción de los atletas es dependiente de la congruencia entre el comportamiento del entrenador percibido y preferido por los deportistas.
\end{abstract}

Palabras clave: Liderazgo, Satisfacción, Psicología del Deporte, Evaluación Psicológica.

A liderança é uma atividade universal evidente nos seres humanos (Bass, 2008) e pode ser definida como o processo comportamental de influenciar indivíduos e grupos para alcançarem determinados objetivos (Barrow, 1977). Sua importância está relacionada ao bom funcionamento e ao aumento da produtividade nas organizações. É um tema de grande interesse dos pesquisadores das ciências sociais, em especial os psicólogos da área organizacional e política (Parry, \& Bryman, 2006).

Estudos sobre liderança têm sido realizados sob a perspectiva de diferentes modelos teóricos e o interesse pelo tema continua crescente (Day, \& Antonakis, 2012; Parry, \& Bryman, 2006). Contudo, o contexto esportivo permanece pouco investigado (Chelladurai, 2012). Isso é surpreendente, tendo em vista que a maior parte das atividades esportivas envolve grupos ou equipes e provavelmente a relação interpessoal entre o treinador e os atletas seja a mais importante neste contexto (Jowet, 2003; Olympiou, Jowett \& Duda, 2008). De fato, nas equipes esportivas o treinador desempenha a função de líder e procura influenciar os atletas para alcançarem seus objetivos. Assim, o comportamento do treinador tem um impacto relevante, de natureza positiva ou negativa, no desempenho dos atletas e no seu bem-estar psicológico (Horn, Bloom, Berglund, \& Packard, 2011). Nessa pers- pectiva, a liderança apresenta-se como um fenômeno de grande importância não só no esporte de alto rendimento, mas também no esporte educacional e lazer.

Até a década de 1970 as investigações foram limitadas pela ausência de um modelo teórico de liderança que contemplasse as especificidades do ambiente esportivo. Por isso, os primeiros estudos sobre a liderança no esporte foram baseados em modelos teóricos de outras áreas, especialmente aqueles provenientes da Psicologia organizacional. Segundo Chelladurai (2012), uma das primeiras iniciativas foi realizada por de Danielson, Zelhart e Drake (1975), que definiram as dimensões do comportamento do líder (treinador) a partir das respostas de atletas escolares a uma versão adaptada do questionário Leadership Behavior Description Questionnaire (Halpin, \&Winer, 1957).

Com o crescente interesse dos pesquisadores pela análise das relações interpessoais no contexto esportivo, Chelladurai (1978) publicou a primeira versão do seu modelo específico para o esporte, denominado Modelo Multidimensional de Liderança (MML). Segundo o próprio autor , o MML foi elaborado a partir dos modelos teóricos existentes para o contexto organizacional, em especial a Teoria da Contingência, a Teoria do Path-Goal, a Teoria Reativa Adaptativa e o Modelo de Discrepância da Liderança (Chelladurai, 
1984). Posteriormente, o MML foi aprimorado (Chelladurai, 1993, 2007) e tem sido a abordagem mais utilizada nos estudos de liderança no esporte nos últimos anos (Aoyagi, Cox, \& McGuire, 2008; Horn et al., 2011).

O MML é uma abordagem interacional baseada em conceitos organizacionais e permite a análise do comportamento do treinador (líder), das variáveis envolvidas e dos resultados desse processo. O comportamento do líder é o componente central do modelo, representado em três estados: requerido (pela situação), preferido (pelos atletas) e atual (Figura 1).

O comportamento requerido diz respeito ao tipo de comportamento esperado pelo sistema organizacional em que se encontra o treinador. Por exemplo, espera-se que o comportamento do treinador seja diferente ao dirigir com uma equipe profissional adulta e ao dirigir uma equipe amadora com atletas adolescentes. Já o comportamento preferido se refere às preferências dos membros do grupo para determinadas formas de comportamento do líder (por exemplo: instrução e orientação, suporte social e feedback). Por sua vez, o comportamento atual indica o comportamento que o treinador realmente exibe, tal como ser atencioso, autoritário ou exigente com as tarefas.

De acordo com o MML, três fatores antecedentes afetam o comportamento do líder: as características da situação, do líder e dos membros do grupo. Características situacionais dizem respeito a fatores do grupo, do esporte e da cultura que influenciam o comportamento requerido. Destacam-se os objetivos, o tipo de esporte (coletivo ou individual, amador ou profissional) e contexto social e cultural do grupo (Chelladurai, 2007, 2012). Estas características situacionais indicam alguns tipos de comportamento que o líder deveria adotar, e também aqueles que deveriam ser evitados.

As características dos membros do grupo incluem a personalidade, nível de habilidade no esporte praticado, sexo e idade. Já as características do líder incluem a sua personalidade, nível de conhecimento e experiência (Chelladurai, 2007, 2012). Observa-se na Figura 1 que tanto o comportamento requerido quanto o preferido são influenciados pelas características situacionais e pelas características dos membros do grupo. Ademais, embora o comportamento atual seja em grande parte determinado pelas características do líder, tal comportamento é também afetado pelas preferências do grupo e pelo contexto (Chelladurai, 2007, 2012).

A principal proposição do MML é que o grau de congruência entre os três estados do comportamento do líder (requerido, preferido e atual) influencia o desempenho e a satisfação dos atletas. Ou seja, a satisfação e o desempenho podem ser aumentados se o comportamento requerido pela situação, o comportamento preferido pelos membros do grupo e o comportamento atual do líder forem semelhantes. Em oposição, se há uma grande diferença entre os três estados do comportamento do líder, a satisfação e o desempenho ficam comprometidos (Andrew, 2009; Chelladurai, 2007, 2012). De forma simplificada, pode-se dizer que a hipótese da congruência pressupõe que, quanto mais o treinador se comportar de acordo com as preferências dos atletas, maior será a satisfação e o desempenho.

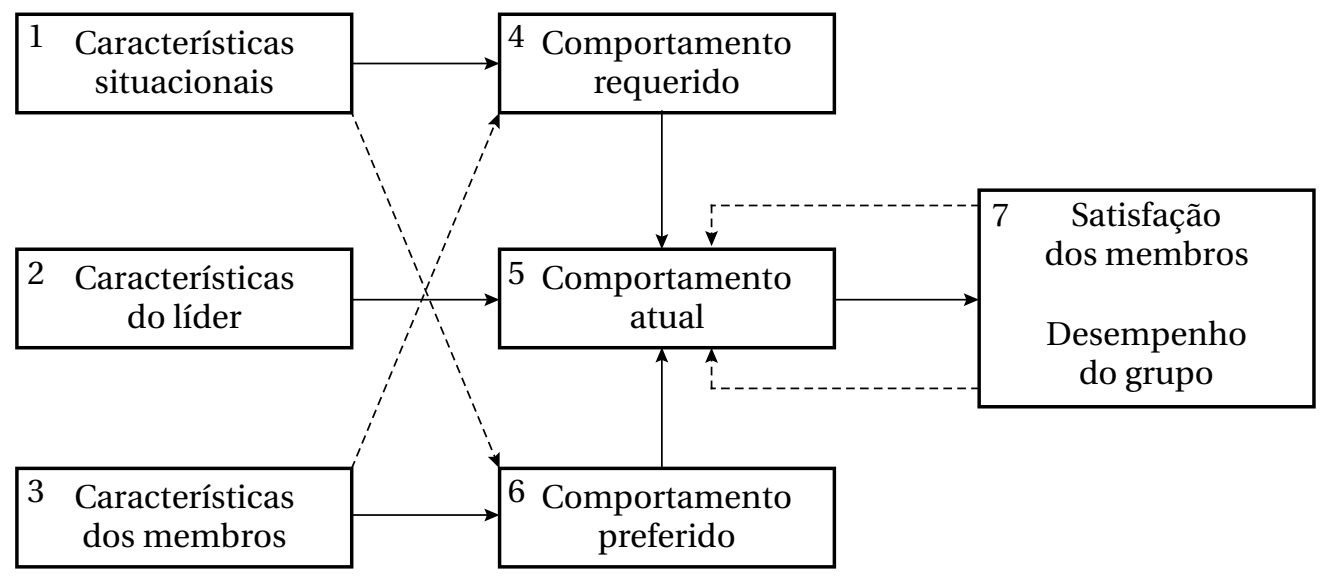

Fonte: Adaptado de Chelladurai (2007).

Figura 1

Modelo Multidimensional de Liderança. 
Essa proposição sugere a necessidade do líder avaliar e regular seu comportamento de acordo com as demandas impostas pela situação e as preferências dos atletas (Chelladurai, 2012). Para isso, o líder deve utilizar os resultados do processo de liderança como referência para ajustar o seu comportamento. Nesse caso, o feedback proveniente da satisfação e desempenho atua como moderador para o ajuste do comportamento atual, conforme indicado na Figura 1. Por exemplo, se o desempenho do grupo está abaixo do esperado, o líder deve direcionar seu comportamento para a execução de tarefas que promovam o aprimoramento das capacidades dos membros do grupo. Por outro lado, se o líder percebe que há insatisfação com o envolvimento entre os componentes do grupo e o próprio líder, este deveria direcionar seu comportamento para o aprimoramento das relações interpessoais (Chelladurai, 2007, 2012).

Embora seja uma variável resultante no MML, a utilização do desempenho para a avaliação da liderança no esporte apresenta importantes limitações, em função de três aspectos: (1) são suscetíveis a fatores como o desempenho extraordinário do adversário, erro de arbitragem ou situações fortuitas (por exemplo, piso inadequado, iluminação deficiente, dificuldades relacionadas à altitude); (2) em algumas ocasiões, mesmo os perdedores da competição poderiam manifestar-se satisfeitos em relação ao esforço individual e do grupo, ao trabalho em equipe e ao treinamento que receberam, porém os índices de desempenho não são capazes de detectar isso; (3) a análise do processo de liderança apenas pelo desempenho nos jogos (vitória x derrota) é falha porque não representa toda a experiência esportiva do atleta. Os atletas investem a maior parte do tempo e esforço nos treinamentos, em relação aos períodos de disputas. Portanto, é necessário considerar as experiências advindas dos períodos de treinamento (Chelladurai, 2007; Chelladurai, \& Riemer, 1997).

Por tudo isso, a satisfação do atleta tem sido indicada como a variável resultante do processo de liderança mais adequada a ser medida, dentro do contexto esportivo (Chelladurai, 2007, 2012; Chelladurai, \& Riemer, 1997). Ela pode ser definida como um estado afetivo positivo, derivado de uma complexa avaliação das estruturas, dos processos e dos resultados associados com as experiências esportivas. Essa avaliação é baseada na discrepância entre o que o atleta deseja e o que ele percebe que está recebendo dentro dos aspectos psicológico, físico e do meio ambiente externo. Ou seja, quanto menor a disparidade entre o que é vivenciado pelo atleta e os seus padrões de exigência, maior será a satisfação com a experiência esportiva (Chelladurai, \& Riemer, 1997).

Especialmente após a elaboração dos modelos de liderança no esporte, a satisfação do atleta também tem recebido a atenção dos pesquisadores. Em primeiro lugar, porque para muitos treinadores a satisfação e o desempenho estão associados, já que a participação do atleta na prática esportiva é primariamente voluntária. Dessa forma, a satisfação parece ser um pré-requisito para que eles alcancem o máximo potencial (Borrego, Leitão, Alves, Silva, \& Palmi, 2010; Riemer, \& Chelladurai, 1998). Nesse sentido, a efetividade das organizações esportivas depende de sua capacidade de satisfazer as necessidades dos atletas. Além disso, no caso dos atletas profissionais, o estudo da satisfação é tão importante quanto o da satisfação no trabalho, já que eles recebem salários e podem ser considerados funcionários da organização (clube ou equipe) (Riemer \& Chelladurai, 1998).

Embora exista na literatura suporte para as proposições gerais do MML (Chelladurai, 2007), os estudos que testaram a hipótese da congruência não confirmaram sua validade. Por exemplo, Riemer e Chelladurai (1995) e Andrew (2009) analisaram atletas de futebol americano e tenistas, respectivamente, e relataram que a hipótese da congruência foi aceita apenas parcialmente. Por outro lado, Çakioglu (2003), Lopes (2006) e Riemer e Toon (2001), analisaram tenistas, jogadores de futebol e atletas de voleibol, respectivamente, e rejeitaram integralmente a hipótese da congruência. Estes autores argumentaram que os fatores situacionais influenciam a relação entre o comportamento de liderança e a satisfação. Dessa forma, a hipótese da congruência poderia não ser confirmada em alguns contextos. Ademais, a pequena amostra no estudo pode ter contribuído para a ausência de efeito da congruência (Çakioglu, 2003; Riemer, \& Toon, 2001).

Além disso, a maior parte das investigações utilizou amostras de atletas adultos (Chelladurai, 2007, 2012), fato que dificulta a transferência dos resultados para outros subgrupos populacionais, como os atletas escolares. As competições esportivas estudantis acontecem em âmbito nacional desde a década de 1960 (Thompson, 2003), mas nos últimos anos assumiram proporções de grandes eventos em nosso país. Por exemplo, a edição do ano 2012 das Olimpíadas 
Escolares contou com a participação de mais de dois milhões de estudantes de todo o país (Comitê Olímpico Brasileiro [COB], 2013). Além do aumento do número de atletas, frequentemente as competições estudantis têm promovido jovens atletas para o esporte de alto rendimento (COB, 2013). Essa realidade amplia a importância dos estudos sobre a interação entre treinadores e atletas no esporte escolar.

Diante do cenário exposto, o presente estudo teve como objetivo testar a hipótese da congruência, proposta pelo MML, em uma amostra de atletas escolares. Ou seja, testar se a congruência entre o comportamento preferido e percebido pelos atletas aumenta a quantidade de variância explicada da satisfação.

\section{Método}

\section{Lócus}

Os dados foram coletados na Etapa Nacional das Olimpíadas Escolares, considerada o maior evento esportivo estudantil do país. Organizado pelo Comitê Olímpico Brasileiro, este evento contou com a participação de 4.025 atletas, com idade entre 15 e 17 anos, representando 1.217 escolas de todos os estados do Brasil e o Distrito Federal. A Etapa Nacional das Olimpíadas Escolares reúne os atletas e equipes de escolas públicas e particulares que obtiveram o melhor desempenho nas seletivas municipais e estaduais. A partir do ano 2013 o evento passou a se chamar Jogos Escolares da Juventude, mas manteve o mesmo formato de disputa e de organização.

\section{Participantes}

Participaram do estudo 466 atletas de ambos os sexos (idade $=16,16$ anos $\pm 0,79$ ) que disputaram as Olimpíadas Escolares em oito modalidades (Tabela 1).

Entre os atletas, $55,8 \%$ eram do sexo masculino, $62,8 \%$ estudavam em escolas públicas, $64,6 \%$ disputaram modalidades coletivas e 50,3\% já haviam participado de edições anteriores das Olimpíadas Escolares. Em média, os atletas treinavam 4,17 vezes por semana ( $\mathrm{DP}=1,47$ ), com duração de 2,28 horas cada treino ( $\mathrm{DP}=0,8)$. O tempo de experiência em competições esportivas variou entre quatro e 144 meses ( $\mathrm{M}=54,8$ e $\mathrm{DP}=31,5)$. Já o tempo de treinamento com o atual treinador variou entre quatro $\mathrm{e}$ 158 meses $(\mathrm{M}=36,7$ e DP $=26,9)$.

\section{Instrumentos}

Os atletas responderam a Escala de Liderança no Esporte, o Questionário de Satisfação do Atleta e um questionário sociodemográfico (Figura 2).

Tabela 1

Distribuição da amostra por sexo e modalidade esportiva.

\begin{tabular}{|c|c|c|c|c|c|c|c|c|c|}
\hline \multirow{3}{*}{ Sexo } & \multicolumn{9}{|c|}{ Modalidades } \\
\hline & \multicolumn{4}{|c|}{ Individuais } & \multicolumn{4}{|c|}{ Coletivas } & \multirow{2}{*}{ Total } \\
\hline & Atletismo & Natação & Taekwondo & Judô & Futsal & Basquete & Vôlei & Handebol & \\
\hline Masculino & 25 & 19 & 29 & 28 & 32 & 26 & 51 & 50 & 260 \\
\hline Feminino & 32 & 9 & 11 & 12 & 44 & 35 & 39 & 24 & 206 \\
\hline Total & 57 & 28 & 40 & 40 & 76 & 61 & 90 & 74 & 466 \\
\hline
\end{tabular}

Atletas

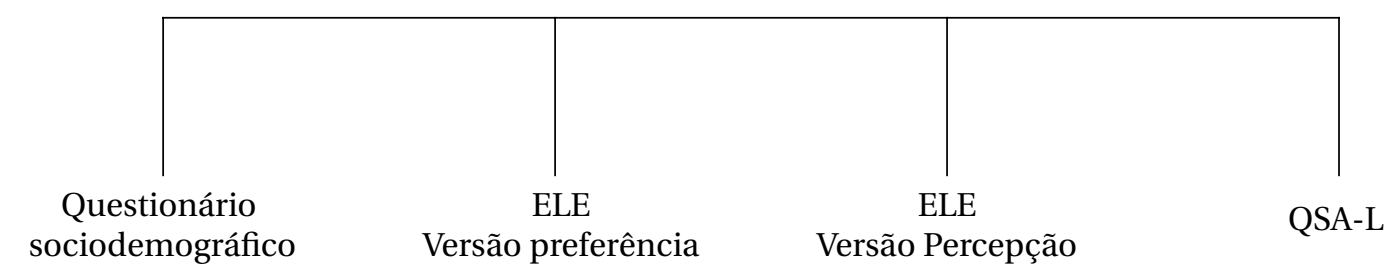

Figura 2

Instrumentos respondidos pelos atletas. 
Escala de Liderança no Esporte (ELE): desenvolvida por Chelladurai e Saleh $(1978 ; 1980)$ e validada no Brasil por Hernandez e Voser (2012). Foi utilizada para determinar o comportamento de liderança preferido pelos atletas (versão preferência) e o comportamento atual dos treinadores (versão percepção). Cada versão possui 40 itens distribuídos nas cinco dimensões da escala: treino e instrução, comportamento democrático, comportamento autocrático, suporte social e feedback positivo. Os itens das duas versões contêm questões idênticas, alterando-se apenas o seu tempo verbal e a frase introdutória: O meu treinador (versão percepção); Eu prefiro que meu treinador (versão preferência). As respostas aos itens da ELE são baseadas na frequência do comportamento exibido pelo treinador e indicadas em uma escala tipo Likert de cinco pontos ( 5 = sempre e $1=$ nunca). $\mathrm{Na}$ versão preferência, os atletas responderam sobre as suas preferências em relação ao comportamento do seu treinador, ou seja, qual o comportamento que ele gostaria que o treinador apresentasse. Na versão percepção, os atletas responderam sobre o comportamento exibido pelo treinador, ou seja, como ele percebe o comportamento habitual do seu treinador.

Questionário de Satisfação do Atleta - Versão Liderança (QSA-L): desenvolvido por Riemer e Chelladurai (1998) e validado no Brasil por Lopes, Samulski e Silva (2007). É composto por 14 itens que representam as quatro dimensões do questionário: desempenho da equipe, desempenho individual, treino e instrução, tratamento pessoal. Os atletas responderam o QSA-L com base na própria avaliação do nível de satisfação com a experiência esportiva. As respostas foram indicadas por meio de uma escala do tipo Likert de sete pontos ( 7 = extremamente e 1 = nunca).

Questionário Sociodemográfico: elaborado especialmente para este trabalho para coletar informações gerais sobre a amostra do estudo. Os atletas informaram a idade, sexo, escola, cidade e estado que representavam na competição, modalidade esportiva e alguns itens relacionados à experiência esportiva (frequência semanal de treinamento, duração de cada treinamento, tempo de experiência em competições esportivas e o tempo de treinamento com o atual treinador).

\section{Procedimentos}

Este estudo foi aprovado pelo Comitê de Ética em Pesquisa da Universidade Federal da Paraíba e respeitou as normas estabelecidas pelo Conselho
Nacional de Saúde (Resolução n ${ }^{\circ}$ 196/96) envolvendo pesquisas com seres humano. A coleta de dados foi realizada nos hotéis onde os atletas estavam hospedados. Antes de responderem os questionários, os participantes assinaram um termo de consentimento livre e esclarecido, demonstrando ciência dos objetivos, procedimentos e liberdade para deixarem de participar da pesquisa quando desejado.

\section{Análise dos dados}

Os dados coletados foram digitados e analisados no programa SPSS (versão 18). O nível de significância adotado nas análises foi de $\mathrm{p} \leq 0,05$. A fim de assegurar a validade e confiabilidade dos dados, inicialmente foi testada a adequação dos instrumentos aos participantes da pesquisa. Para isso, foi utilizada a análise fatorial exploratória (AFE) e a verificação da consistência interna pelo coeficiente alfa de Cronbach.

Para testar a hipótese da congruência entre a liderança preferida e percebida com a satisfação, os procedimentos adotados foram baseados nas sugestões de Chelladurai $(2007 ; 2012)$ e em outros estudos similares (Andrew, 2004; 2009; Chelladurai, \& Riemer, 1998; Lopes, 2006; Riemer, e Chelladurai, 1995; Riemer, \& Toon, 2001). Foram realizadas cinco análises de regressão linear múltipla (método enter por blocos) para cada uma das quatro dimensões da satisfação (treino e instrução, tratamento pessoal, desempenho da equipe e desempenho individual), totalizando 20 análises. Foi inserido primeiro o valor da preferência, seguido pelo valor da percepção e do termo de interação (preferência, percepção, interação). O termo de interação foi obtido a partir da multiplicação do valor da preferência pelo valor da percepção (interação = preferência x percepção) em cada uma das cinco dimensões da liderança. A hipótese da congruência foi considerada válida se a variável interação tiver aumentado significativamente a explicação da variância na satisfação, demonstrado pelo $\Delta R^{2}$ (variação no coeficiente de determinação).

\section{Resultados}

\section{Adequação dos instrumentos aos participantes}

A ELE derivada da AFE confirmou parcialmente a sua estrutura original. Apenas cinco itens da escala se 
apresentaram em um fator diferente daquele proposto por Chelladurai e Saleh (1980). Já o QSA-L derivado da AFE resultou em uma estrutura idêntica à original. $\mathrm{Ou}$ seja, a distribuição dos itens pelos quatro fatores não diferiu daquela apresentada por Riemer e Chelladurai (1998). Já o $\alpha$ Cronbach variou entre 0,68 (comportamento democrático) e 0,87 (treino e instrução) para a ELE, e entre 0,74 (desempenho da equipe) e 0,86 (tratamento pessoal) para o QSA-L.

\section{Teste da hipótese da congruência}

A primeira dimensão da satisfação avaliada foi o treino e instrução (Tabela 2). Observa-se aumento significativo da variância explicada com a inserção da variável interação nas seguintes dimensões da liderança: treino e instrução, comportamento democrático e comportamento autocrático. Apenas para esses casos a hipótese da congruência foi aceita, sendo rejeitada nas outras dimensões (suporte social e feedback

Tabela 2

Variância da satisfação com o Treino e Instrução atribuída à liderança.

\begin{tabular}{|c|c|c|c|c|c|c|c|}
\hline \multirow{2}{*}{$\begin{array}{l}\text { Dimensão da } \\
\text { liderança }\end{array}$} & \multirow[b]{2}{*}{ Modelo } & \multirow[b]{2}{*}{ Preditor } & \multicolumn{3}{|c|}{ Beta $(\beta)$} & \multicolumn{2}{|c|}{ Variância } \\
\hline & & & $\begin{array}{c}\text { Não } \\
\text { Padronizado }\end{array}$ & Padronizado & $\mathrm{p}$ & $\mathrm{R}^{2}$ & $\Delta \mathrm{R}^{2}$ \\
\hline \multirow{3}{*}{ TI } & 1 & Preferência & 0,562 & 0,340 & $<0,001$ & 0,116 & $0,116^{*}$ \\
\hline & 2 & $\begin{array}{l}\text { Preferência } \\
\text { Percepção }\end{array}$ & $\begin{array}{l}-0,006 \\
0,921\end{array}$ & $\begin{array}{c}-0,003 \\
0,611\end{array}$ & $\begin{array}{c}0,938 \\
<0,001\end{array}$ & 0,371 & $0,255^{*}$ \\
\hline & 3 & $\begin{array}{l}\text { Preferência } \\
\text { Percepção } \\
\text { Interação }\end{array}$ & $\begin{array}{c}-1,218 \\
-0,339 \\
0,284\end{array}$ & $\begin{array}{c}-0,737 \\
-0,225 \\
1,392\end{array}$ & $\begin{array}{l}0,005 \\
0,450 \\
0,005\end{array}$ & 0,382 & $0,011^{*}$ \\
\hline \multirow{3}{*}{ CD } & 1 & Preferência & 0,136 & 0,114 & 0,014 & 0,013 & $0,013^{*}$ \\
\hline & 2 & $\begin{array}{l}\text { Preferência } \\
\text { Percepção }\end{array}$ & $\begin{array}{c}-0,018 \\
0,265\end{array}$ & $\begin{array}{c}-0,015 \\
0,247\end{array}$ & $\begin{array}{c}0,781 \\
<0,001\end{array}$ & 0,058 & $0,045^{*}$ \\
\hline & 3 & $\begin{array}{l}\text { Preferência } \\
\text { Percepção } \\
\text { Interação }\end{array}$ & $\begin{array}{c}-0,620 \\
-0,398 \\
0,182\end{array}$ & $\begin{array}{c}-0,519 \\
-0,371 \\
0,988\end{array}$ & $\begin{array}{l}0,007 \\
0,111 \\
0,006\end{array}$ & 0,073 & $0,015^{*}$ \\
\hline \multirow{3}{*}{ CA } & 1 & Preferência & $-0,027$ & $-0,042$ & 0,365 & 0,002 & 0,002 \\
\hline & 2 & $\begin{array}{l}\text { Preferência } \\
\text { Percepção }\end{array}$ & $\begin{array}{l}-0,115 \\
0,204\end{array}$ & $\begin{array}{l}-0,182 \\
0,287\end{array}$ & $\begin{array}{l}<0,001 \\
<0,001\end{array}$ & 0,064 & $0,063^{*}$ \\
\hline & 3 & $\begin{array}{l}\text { Preferência } \\
\text { Percepção } \\
\text { Interação }\end{array}$ & $\begin{array}{c}-0,608 \\
-0,303 \\
0,153\end{array}$ & $\begin{array}{c}-0,957 \\
-0,425 \\
1,310\end{array}$ & $\begin{array}{c}<0,001 \\
0,001 \\
<0,001\end{array}$ & 0,134 & $0,070^{*}$ \\
\hline \multirow{3}{*}{ SS } & 1 & Preferência & 0,357 & 0,344 & $<0,001$ & 0,118 & $0,118^{*}$ \\
\hline & 2 & $\begin{array}{l}\text { Preferência } \\
\text { Percepção }\end{array}$ & $\begin{array}{l}0,187 \\
0,279\end{array}$ & $\begin{array}{l}0,180 \\
0,277\end{array}$ & $\begin{array}{c}0,001 \\
<0,001\end{array}$ & 0,168 & $0,050^{*}$ \\
\hline & 3 & $\begin{array}{l}\text { Preferência } \\
\text { Percepçãao } \\
\text { Interação }\end{array}$ & $\begin{array}{l}0,058 \\
0,145 \\
0,034\end{array}$ & $\begin{array}{l}0,056 \\
0,144 \\
0,231\end{array}$ & $\begin{array}{l}0,791 \\
0,519 \\
0,542\end{array}$ & 0,169 & 0,001 \\
\hline \multirow{3}{*}{$\mathrm{FP}$} & 1 & Preferência & 0.380 & 0,322 & $<0,001$ & 0,104 & $0,104^{*}$ \\
\hline & 2 & $\begin{array}{l}\text { Preferência } \\
\text { Percepção }\end{array}$ & $\begin{array}{l}0,237 \\
0,268\end{array}$ & $\begin{array}{l}0,201 \\
0,234\end{array}$ & $\begin{array}{l}<0,001 \\
<0,001\end{array}$ & 0,144 & $0,040^{*}$ \\
\hline & 3 & $\begin{array}{l}\text { Preferência } \\
\text { Percepção } \\
\text { Interação }\end{array}$ & $\begin{array}{c}-0,192 \\
-0,191 \\
0,106\end{array}$ & $\begin{array}{l}-0,163 \\
-0,166 \\
0,670\end{array}$ & $\begin{array}{l}0,499 \\
0,529 \\
0,124\end{array}$ & 0,148 & 0,004 \\
\hline
\end{tabular}

TI: Treino e Instrução; CD: Comportamento Democrático; CA: Comportamento Autocrático; SS: Suporte Social; FP: Feedback Positivo; $\mathrm{R}^{2}$ : Coeficiente de determinação; $\Delta \mathrm{R}^{2}$ : Variação no coeficiente de determinação.

*Aumento significativo na variância explicada $(\mathrm{p}<0,05)$. 
positivo). Dentre as dimensões da liderança, percebe-se que o treino e instrução foi o que apresentou o maior $\mathrm{R}^{2} \mathrm{e}$, portanto, o que mais contribuiu individualmente para a explicação da variância da satisfação com o treino e instrução.

A segunda dimensão da satisfação avaliada foi o tratamento pessoal (Tabela 3). Observa-se aumento significativo da variância explicada com a inserção da variável interação apenas nas dimensões comportamento autocrático e feedback positivo. Portanto, apenas para essas dimensões a hipótese da congruência foi aceita. O treino e instrução, suporte social e feedback positivo apresentaram os maiores $\mathrm{R}^{2} \mathrm{e}$, assim, foram os que mais contribuíram individualmente para a explicação da variância na satisfação com tratamento pessoal.

A terceira dimensão da satisfação a ser avaliada foi o desempenho da equipe (Tabela 4). A inserção da variável interação não provocou aumento significativo da variância explicada em nenhuma das dimen-

Tabela 3

Variância da satisfação com o Tratamento Pessoal atribuída à liderança.

\begin{tabular}{|c|c|c|c|c|c|c|c|}
\hline \multirow{2}{*}{$\begin{array}{l}\text { Dimensão da } \\
\text { liderança }\end{array}$} & \multirow[b]{2}{*}{ Modelo } & \multirow[b]{2}{*}{ Preditor } & \multicolumn{3}{|c|}{ Beta $(\beta)$} & \multicolumn{2}{|c|}{ Variância } \\
\hline & & & $\begin{array}{c}\text { Não } \\
\text { Padronizado }\end{array}$ & Padronizado & $\mathrm{p}$ & $\mathrm{R}^{2}$ & $\Delta \mathrm{R}^{2}$ \\
\hline \multirow{3}{*}{$\mathrm{TI}$} & 1 & Preferência & 0,559 & 0,336 & $<0,001$ & 0.113 & $0,113^{*}$ \\
\hline & 2 & $\begin{array}{l}\text { Preferência } \\
\text { Percepção }\end{array}$ & $\begin{array}{l}0,145 \\
0,670\end{array}$ & $\begin{array}{l}0,087 \\
0,442\end{array}$ & $\begin{array}{c}0,074 \\
<0,001\end{array}$ & 0.246 & $0,133^{*}$ \\
\hline & 3 & $\begin{array}{l}\text { Preferência } \\
\text { Percepção } \\
\text { Interação }\end{array}$ & $\begin{array}{l}-0,425 \\
0,077 \\
0,134\end{array}$ & $\begin{array}{l}-0,256 \\
0,051 \\
0,651\end{array}$ & $\begin{array}{l}0,376 \\
0,877 \\
0,228\end{array}$ & 0.248 & 0,002 \\
\hline \multirow{3}{*}{ CD } & 1 & Preferência & 0,274 & 0,228 & $<0,001$ & 0.052 & $0,052^{*}$ \\
\hline & 2 & $\begin{array}{l}\text { Preferência } \\
\text { Percepção }\end{array}$ & $\begin{array}{l}0,049 \\
0,389\end{array}$ & $\begin{array}{l}0,041 \\
0,360\end{array}$ & $\begin{array}{c}0,420 \\
<0,001\end{array}$ & 0.146 & $0,095^{*}$ \\
\hline & 3 & $\begin{array}{l}\text { Preferência } \\
\text { Percepção } \\
\text { Interação }\end{array}$ & $\begin{array}{c}0,361 \\
0,733 \\
-0,095\end{array}$ & $\begin{array}{c}0,300 \\
0,678 \\
-0,509\end{array}$ & $\begin{array}{l}0,103 \\
0,002 \\
0,142\end{array}$ & 0.150 & 0,004 \\
\hline \multirow{3}{*}{ CA } & 1 & Preferência & $-0,025$ & -0.040 & 0,392 & 0.002 & 0,002 \\
\hline & 2 & $\begin{array}{l}\text { Preferência } \\
\text { Percepção }\end{array}$ & $\begin{array}{l}-0,129 \\
0,238\end{array}$ & $\begin{array}{c}-0,202 \\
0,333\end{array}$ & $\begin{array}{l}<0,001 \\
<0,001\end{array}$ & 0.086 & $0,084^{*}$ \\
\hline & 3 & $\begin{array}{l}\text { Preferência } \\
\text { Percepção } \\
\text { Interação }\end{array}$ & $\begin{array}{c}-0,626 \\
-0,272 \\
0,154\end{array}$ & $\begin{array}{c}-0,978 \\
-0,380 \\
1,311\end{array}$ & $\begin{array}{c}<0,001 \\
0,003 \\
<0,001\end{array}$ & 0.156 & $0,070 *$ \\
\hline \multirow{3}{*}{ SS } & 1 & Preferência & 0,458 & 0,438 & $<0,001$ & 0.192 & $0,192^{*}$ \\
\hline & 2 & $\begin{array}{l}\text { Preferência } \\
\text { Percepção }\end{array}$ & $\begin{array}{l}0,279 \\
0,294\end{array}$ & $\begin{array}{l}0,267 \\
0,290\end{array}$ & $\begin{array}{l}<0,001 \\
<0,001\end{array}$ & 0.247 & $0,055^{*}$ \\
\hline & 3 & $\begin{array}{l}\text { Preferência } \\
\text { Percepção } \\
\text { Interação }\end{array}$ & $\begin{array}{r}0,436 \\
0,456 \\
-0,041\end{array}$ & $\begin{array}{c}0,417 \\
0,450 \\
-0,278\end{array}$ & $\begin{array}{l}0,037 \\
0,035 \\
0,439\end{array}$ & 0.248 & 0,001 \\
\hline \multirow{3}{*}{ FP } & 1 & Preferência & 0,456 & 0,384 & $<0,001$ & 0.147 & $0,147^{*}$ \\
\hline & 2 & $\begin{array}{l}\text { Preferência } \\
\text { Percepção }\end{array}$ & $\begin{array}{l}0,229 \\
0,423\end{array}$ & $\begin{array}{l}0,193 \\
0,366\end{array}$ & $\begin{array}{l}<0,001 \\
<0,001\end{array}$ & 0.245 & $0,098^{*}$ \\
\hline & 3 & $\begin{array}{l}\text { Preferência } \\
\text { Percepção } \\
\text { Interação }\end{array}$ & $\begin{array}{c}-0,549 \\
-0,408 \\
0,192\end{array}$ & $\begin{array}{c}-0,462 \\
-0,354 \\
1,205\end{array}$ & $\begin{array}{l}0,040 \\
0,152 \\
0,003\end{array}$ & 0.259 & $0,014^{*}$ \\
\hline
\end{tabular}

TI: Treino e Instrução; CD: Comportamento Democrático; CA: Comportamento Autocrático; SS: Suporte Social; FP: Feedback Positivo; $\mathrm{R}^{2}$ : Coeficiente de determinação; $\Delta \mathrm{R}^{2}$ : Variação no coeficiente de determinação.

*Aumento significativo na variância explicada $(\mathrm{p}<0,05)$. 
sões da liderança. Portanto, não há suporte para hipótese da congruência em relação à satisfação com o desempenho da equipe.

A quarta e última dimensão da satisfação a ser avaliada foi o desempenho individual (Tabela 5). Observa-se aumento significativo da variância explicada com a inserção da variável interação nas dimensões comportamento autocrático, suporte social e feedback positivo. Assim, para essas dimen- sões a hipótese da congruência foi aceita, sendo rejeitada nas demais.

Estes resultados suportam parcialmente a hipótese de que a satisfação dos atletas é dependente da congruência entre a liderança percebida e preferida pelos atletas, já que a inserção do termo interação aumentou significativamente a explicação da variância na satisfação em três das quatro dimensões (treino e instrução, tratamento pessoal e desempenho individual).

Tabela 4

Variância da satisfação com o Desempenho da Equipe atribuída à liderança.

\begin{tabular}{|c|c|c|c|c|c|c|c|}
\hline \multirow{2}{*}{$\begin{array}{l}\text { Dimensão da } \\
\text { liderança }\end{array}$} & \multirow[b]{2}{*}{ Modelo } & \multirow[b]{2}{*}{ Preditor } & \multicolumn{3}{|c|}{ Beta $(\beta)$} & \multicolumn{2}{|c|}{ Variância } \\
\hline & & & $\begin{array}{c}\text { Não } \\
\text { Padronizado }\end{array}$ & Padronizado & $\mathrm{p}$ & $\mathrm{R}^{2}$ & $\Delta \mathrm{R}^{2}$ \\
\hline \multirow{3}{*}{ TI } & 1 & Preferência & 0,114 & 0,055 & 0,234 & 0.003 & 0,003 \\
\hline & 2 & $\begin{array}{l}\text { Preferência } \\
\text { Percepção }\end{array}$ & $\begin{array}{c}-0,183 \\
0,481\end{array}$ & $\begin{array}{c}-0,089 \\
0,256\end{array}$ & $\begin{array}{c}0,107 \\
<0,001\end{array}$ & 0.048 & $0,045^{*}$ \\
\hline & 3 & $\begin{array}{l}\text { Preferência } \\
\text { Percepção } \\
\text { Interação }\end{array}$ & $\begin{array}{c}-0,850 \\
-0,212 \\
0,156\end{array}$ & $\begin{array}{c}-0,412 \\
-0,113 \\
0,614\end{array}$ & $\begin{array}{l}0,204 \\
0,759 \\
0,312\end{array}$ & 0.050 & $0,045^{*}$ \\
\hline \multirow{3}{*}{ CD } & 1 & Preferência & 0,197 & 0,132 & 0,004 & 0.017 & $0,017^{*}$ \\
\hline & 2 & $\begin{array}{l}\text { Preferência } \\
\text { Percepção }\end{array}$ & $\begin{array}{l}0,164 \\
0,056\end{array}$ & $\begin{array}{l}0,110 \\
0,042\end{array}$ & $\begin{array}{l}0,041 \\
0,438\end{array}$ & 0.019 & 0,001 \\
\hline & 3 & $\begin{array}{l}\text { Preferência } \\
\text { Percepção } \\
\text { Interação }\end{array}$ & $\begin{array}{c}-0,379 \\
-0,541 \\
0,164\end{array}$ & $\begin{array}{c}-0,254 \\
-0,405 \\
0,713\end{array}$ & $\begin{array}{l}0,197 \\
0,090 \\
0,055\end{array}$ & 0.027 & 0,008 \\
\hline \multirow{3}{*}{ CA } & 1 & Preferência & $-0,121$ & $-0,153$ & 0,001 & 0.023 & $0,023^{*}$ \\
\hline & 2 & $\begin{array}{l}\text { Preferência } \\
\text { Percepção }\end{array}$ & $\begin{array}{c}-0,142 \\
0,049\end{array}$ & $\begin{array}{l}-0,180 \\
0,056\end{array}$ & $\begin{array}{l}0,001 \\
0,291\end{array}$ & 0.026 & $0,023^{*}$ \\
\hline & 3 & $\begin{array}{l}\text { Preferência } \\
\text { Percepção } \\
\text { Interação }\end{array}$ & $\begin{array}{c}-0,341 \\
-0,155 \\
0,061\end{array}$ & $\begin{array}{l}-0,430 \\
-0,174 \\
0,423\end{array}$ & $\begin{array}{l}0,003 \\
0,194 \\
0,063\end{array}$ & 0.033 & 0,002 \\
\hline \multirow{3}{*}{ SS } & 1 & Preferência & 0,280 & 0,216 & $<0,001$ & 0.047 & $0,047^{*}$ \\
\hline & 2 & $\begin{array}{l}\text { Preferência } \\
\text { Percepção }\end{array}$ & $\begin{array}{l}0,279 \\
0,001\end{array}$ & $\begin{array}{l}0,216 \\
0,001\end{array}$ & $\begin{array}{c}<0,001 \\
0,987\end{array}$ & 0.047 & 0 \\
\hline & 3 & $\begin{array}{l}\text { Preferência } \\
\text { Percepção } \\
\text { Interação }\end{array}$ & $\begin{array}{c}-0,185 \\
-0,479 \\
0,121\end{array}$ & $\begin{array}{c}-0,143 \\
-0,382 \\
0,666\end{array}$ & $\begin{array}{l}0,524 \\
0,110 \\
0,099\end{array}$ & 0.052 & 0,006 \\
\hline \multirow{3}{*}{ FP } & 1 & Preferência & 0,154 & 0,104 & 0,024 & 0,011 & $0,011^{*}$ \\
\hline & 2 & $\begin{array}{l}\text { Preferência } \\
\text { Percepção }\end{array}$ & $\begin{array}{l}0,089 \\
0,121\end{array}$ & $\begin{array}{l}0,060 \\
0,085\end{array}$ & $\begin{array}{l}0,265 \\
0,117\end{array}$ & 0.016 & 0,005 \\
\hline & 3 & $\begin{array}{l}\text { Preferência } \\
\text { Percepção } \\
\text { Interação }\end{array}$ & $\begin{array}{c}-0,503 \\
-0,511 \\
0,146\end{array}$ & $\begin{array}{c}-0,342 \\
-0,357 \\
0,740\end{array}$ & $\begin{array}{l}0,186 \\
0,207 \\
0,112\end{array}$ & 0.022 & 0,005 \\
\hline
\end{tabular}

TI: Treino e Instrução; CD: Comportamento Democrático; CA: Comportamento Autocrático; SS: Suporte Social; FP: Feedback Positivo; $\mathrm{R}^{2}$ : Coeficiente de determinação; $\Delta \mathrm{R}^{2}$ : Variação no coeficiente de determinação.

*Aumento significativo na variância explicada $(\mathrm{p}<0,05)$. 
Tabela 5

Variância da satisfação com o Desempenho Individual atribuída à liderança.

\begin{tabular}{|c|c|c|c|c|c|c|c|}
\hline \multirow{2}{*}{$\begin{array}{l}\text { Dimensão da } \\
\text { liderança }\end{array}$} & \multirow[b]{2}{*}{ Modelo } & \multirow[b]{2}{*}{ Preditor } & \multicolumn{3}{|c|}{ Beta $(\beta)$} & \multicolumn{2}{|c|}{ Variância } \\
\hline & & & $\begin{array}{c}\text { Não } \\
\text { Padronizado }\end{array}$ & Padronizado & $\mathrm{p}$ & $\mathrm{R}^{2}$ & $\Delta \mathrm{R}^{2}$ \\
\hline \multirow{3}{*}{ TI } & 1 & Preferência & 0,281 & 0,141 & 0,002 & 0.020 & $0,020^{*}$ \\
\hline & 2 & $\begin{array}{l}\text { Preferência } \\
\text { Percepção }\end{array}$ & $\begin{array}{l}-0,009 \\
0,471\end{array}$ & $\begin{array}{c}-0,005 \\
0,259\end{array}$ & $\begin{array}{c}0,932 \\
<0,001\end{array}$ & 0.066 & $0,046^{*}$ \\
\hline & 3 & $\begin{array}{l}\text { Preferência } \\
\text { Percepção } \\
\text { Interação }\end{array}$ & $\begin{array}{c}-0,884 \\
-0,439 \\
0,205\end{array}$ & $\begin{array}{c}-0,444 \\
-0,241 \\
0,834\end{array}$ & $\begin{array}{l}0,167 \\
0,508 \\
0,165\end{array}$ & 0.070 & 0,004 \\
\hline \multirow{3}{*}{$\mathrm{CD}$} & 1 & Preferência & 0.220 & 0,152 & 0,001 & 0.023 & $0,023^{*}$ \\
\hline & 2 & $\begin{array}{l}\text { Preferência } \\
\text { Percepção }\end{array}$ & $\begin{array}{l}0,146 \\
0,128\end{array}$ & $\begin{array}{l}0,101 \\
0,099\end{array}$ & $\begin{array}{l}0,059 \\
0,066\end{array}$ & 0.030 & 0,007 \\
\hline & 3 & $\begin{array}{l}\text { Preferência } \\
\text { Percepção } \\
\text { Interação }\end{array}$ & $\begin{array}{l}0,035 \\
0,005 \\
0,034\end{array}$ & $\begin{array}{l}0,024 \\
0,004 \\
0,151\end{array}$ & $\begin{array}{l}0,902 \\
0,986 \\
0,683\end{array}$ & 0.031 & 0 \\
\hline \multirow{3}{*}{ CA } & 1 & Preferência & $-0,109$ & $-0,142$ & 0,002 & 0.020 & $0,020^{*}$ \\
\hline & 2 & $\begin{array}{l}\text { Preferência } \\
\text { Percepção }\end{array}$ & $\begin{array}{c}-0,181 \\
0,166\end{array}$ & $\begin{array}{c}-0,236 \\
0,193\end{array}$ & $\begin{array}{l}<0,001 \\
<0,001\end{array}$ & 0.049 & $0,028^{*}$ \\
\hline & 3 & $\begin{array}{l}\text { Preferência } \\
\text { Percepção } \\
\text { Interação }\end{array}$ & $\begin{array}{c}-0,532 \\
-0,195 \\
0,109\end{array}$ & $\begin{array}{c}-0,696 \\
-0,228 \\
0,775\end{array}$ & $\begin{array}{c}<0,001 \\
<0,083 \\
0,001\end{array}$ & 0.073 & $0,024^{*}$ \\
\hline \multirow{3}{*}{ SS } & 1 & Preferência & 0,281 & 0,225 & $<0,001$ & 0.050 & $0,050^{*}$ \\
\hline & 2 & $\begin{array}{l}\text { Preferência } \\
\text { Percepção }\end{array}$ & $\begin{array}{l}0,204 \\
0,126\end{array}$ & $\begin{array}{l}0,163 \\
0,104\end{array}$ & $\begin{array}{l}0,004 \\
0,064\end{array}$ & 0.057 & 0,007 \\
\hline & 3 & $\begin{array}{l}\text { Preferência } \\
\text { Percepção } \\
\text { Interação }\end{array}$ & $\begin{array}{c}-0,570 \\
-0,676 \\
0,201\end{array}$ & $\begin{array}{c}-0,456 \\
-0,558 \\
1,149\end{array}$ & $\begin{array}{l}0,040 \\
0,019 \\
0,004\end{array}$ & 0.074 & $0,017^{*}$ \\
\hline \multirow{3}{*}{ FP } & 1 & Preferência & 0,187 & 0,132 & 0,004 & 0.017 & $0,017^{*}$ \\
\hline & 2 & $\begin{array}{l}\text { Preferência } \\
\text { Percepção }\end{array}$ & $\begin{array}{l}0,039 \\
0,276\end{array}$ & $\begin{array}{l}0,027 \\
0,200\end{array}$ & $\begin{array}{c}0,609 \\
<0,001\end{array}$ & 0.046 & $0,029 *$ \\
\hline & 3 & $\begin{array}{l}\text { Preferência } \\
\text { Percepção } \\
\text { Interação }\end{array}$ & $\begin{array}{c}-0,776 \\
-0,594 \\
0,202\end{array}$ & $\begin{array}{c}-0,546 \\
-0,430 \\
1,055\end{array}$ & $\begin{array}{l}0,032 \\
0,122 \\
0,021\end{array}$ & 0.057 & $0,011^{*}$ \\
\hline
\end{tabular}

TI: Treino e Instrução; CD: Comportamento Democrático; CA: Comportamento Autocrático; SS: Suporte Social; FP: Feedback Positivo; $\mathrm{R}^{2}$ : Coeficiente de determinação; $\Delta \mathrm{R}^{2}$ : Variação no coeficiente de determinação.

*Aumento significativo na variância explicada $(\mathrm{p}<0,05)$.

\section{Discussão}

O presente estudo realizou uma série de análises de regressão para testar a hipótese de que a satisfação é dependente da congruência entre a liderança preferida e percebida pelos atletas. Esta hipótese foi confirmada para três dimensões da satisfação: treino e instrução (TI), tratamento pessoal (TP) e desempenho da individual (DI). Porém, foi rejeitada para a satisfação com o desempenho da equipe (DE).
Em relação à satisfação com o TI, a congruência entre a liderança preferida e percebida nas dimensões TI, Comportamento Democrático (CD) e Comportamento Autocrático (CA) resultaram em aumento significativo no $\mathrm{R}^{2}$. Portanto, se os atletas perceberem que estão recebendo o nível desejado desses comportamentos, sua satisfação com o treinamento e as instruções fornecidas pelo treinador será aumentada. Já na satisfação com o TP, 
a congruência entre a liderança preferida e percebida nas dimensões CA e Feedback Positivo (FP) resultaram em aumento significativo no $\mathrm{R}^{2}$. Portanto, se os atletas perceberem que estão recebendo o nível desejado desses comportamentos, sua satisfação com os relacionamentos interpessoais no grupo e o incentivo/reconhecimento fornecido pelo treinador será aumentada.

Por último, em relação à satisfação com o DI, a congruência entre a liderança preferida e percebida nas dimensões CA, Suporte Social (SS) e FP resultaram em aumento significativo no $\mathrm{R}^{2}$. Portanto, se os atletas perceberem que estão recebendo o nível desejado desses comportamentos, sua satisfação com o próprio desempenho será aumentada. Contudo, na satisfação com o DE, a congruência entre a liderança preferida e percebida não provocou mudança significativa no $\mathrm{R}^{2}$. Portanto, não há suporte para a hipótese da congruência em relação à satisfação do atleta com o nível de desempenho da sua equipe.

Analisando estes resultados sob o ponto de vista dos comportamentos de liderança, observa-se que todas as cinco dimensões da ELE tiveram impacto significativo na satisfação dos atletas. Ou seja, a congruência entre a liderança preferida e percebida de todas as dimensões da liderança aumentou a variância explicada de pelo menos uma dimensão da satisfação dos atletas. O CA influenciou a satisfação com o treino e instrução, com o tratamento pessoal e com o desempenho individual. Já o FP influenciou a satisfação como tratamento pessoal e o desempenho individual. Por sua vez, o TI e o CD influenciaram apenas a satisfação com treino e instrução. Por fim, o SS influenciou apenas a satisfação com o desempenho individual (Tabela 6).

Conforme a Tabela 6 , a congruência do CA é o que mais influenciou a satisfação dos atletas. Essa é uma constatação interessante, pois, embora o comportamento autocrático seja o menos desejado pelos atletas (Rodrigues, 2014), a sua congruência foi a que mais influenciou a satisfação. Portanto, o nível de CA que o treinador fornece e a percepção dos atletas sobre tal comportamento é de grande relevância para a experiência esportiva dos atletas.

Contudo, observa-se que a literatura tem apresentado resultados inconsistentes em relação à hipótese da congruência do MML. De acordo com Chelladurai e seus colaboradores (Chelladurai, \& Riemer, 1998; Riemer, \& Chelladurai, 1995; Riemer, \& Toon, 2001), as
Tabela 6

Dimensões da liderança que influenciaram a satisfação dos atletas, de acordo com o teste da Hipótese da Congruência.

\begin{tabular}{ll}
\hline Liderança & Satisfação \\
\hline & Treino e Instrução \\
CA & Tratamento Pessoal \\
& Desempenho Individual \\
& Tratamento Pessoal \\
FP & Desempenho Individual \\
TI & Treino e Instrução \\
CD & Treino e Instrução \\
SS & Desempenho Individual \\
\hline
\end{tabular}

CA: Comportamento Autocrático; FP: Feedback Positivo; TI: Treino e Instrução; CD: Comportamento Democrático; SS: Suporte Social.

investigações iniciais foram realizadas de forma inadequada. Nesses estudos (Chelladurai, 1984; Horne, \& Carron, 1985), os pesquisadores criaram uma variável independente híbrida, derivada da discrepância entre dois tipos de comportamento (por exemplo, subtraindo as pontuações do comportamento percebido pelo preferido). Posteriormente, essa nova variável foi associada ao desempenho e satisfação dos atletas para verificar a hipótese da congruência. Esse procedimento gerou problemas na validade e confiabilidade dos dados, assim como produziu correlações espúrias (Andrew, 2009; Chelladurai, \& Riemer, 1998; Riemer, \& Chelladurai, 1995). Além disso, esses estudos utilizaram uma questão única para medir a satisfação dos atletas, o que também compromete a sua validade.

Portanto, embora intuitivamente atraente, a utilização dos escores derivados da discrepância entre as variáveis mostrou-se problemática (Riemer, \& Toon, 2001). Uma solução para essa questão foi sugerida por Riemer e Chelladurai (1995). Segundo esses pesquisadores, Cronbach (1958 apud Riemer, \& Chelladurai, 1995) demonstrou que a interação entre dois componentes (por exemplo, preferência x percepção) é equivalente à diferença entre esses mesmos componentes. Assim, para evitar os problemas gerados com a utilização de escores derivados da discrepância entre duas variáveis (por exemplo, preferência - percepção), dever-se-ia testar a hipótese da congruência por meio da análise de regressão linear múltipla, com as variáveis medidas e a interação entre elas. Nesse proce- 
dimento, os componentes principais (preferência e percepção) devem ser inseridos primeiramente na regressão, seguidos pela resultante da interação entre eles (preferência x percepção). Esse procedimento permite testar se a interação aumenta a variância explicada pelos componentes principais. Dessa forma, a hipótese da congruência é considerada válida se a interação entre os termos (preferência $x$ percepção) aumentar significativamente a quantidade de variância explicada.

Este foi o método utilizado pelo presente estudo e por outras investigações (Andrew, 2004; 2009; Çakioglu, 2003; Chelladurai, \& Riemer, 1998; Lopes, 2006; Riemer, \& Chelladurai,1995; Riemer, \& Toon, 2001) e tem sido considerado adequado para testar a hipótese da congruência. Não obstante, ainda há inconsistência entre os resultados desses estudos. Por exemplo, Riemer e Chelladurai (1995) avaliaram o impacto da congruência entre a liderança preferida e percebida na satisfação de atletas de futebol americano. Os autores relataram que a hipótese da congruência foi aceita apenas para a dimensão suporte social. Além disso, em relação às dimensões treino e instrução e feedback positivo, as percepções dos atletas foram melhores preditores da satisfação quando comparado com a preferência e a congruência. Por outro lado, Riemer e Toon (2001) e Çakioglu (2003) analisaram tenistas e jogadores de futebol, respectivamente, e rejeitaram a hipótese da congruência para todas as dimensões da liderança.

Embora Riemer e Toon (2001) e Çakioglu (2003) tenham utilizado os mesmos instrumentos do presente estudo (ELE e QSA-L), o contraste entre os resultados pode ser devido a diversas diferenças entre as investigações. O MML sustenta que as características situacionais e dos membros do grupo exercem influências significativas no processo de liderança. Por exemplo, já foi mostrado que a idade, a nacionalidade, o sexo e o tipo da modalidade afetam as preferências dos atletas pela liderança (Chelladurai, \& Carron, 1983; Hernandez, \& Voser, 2012; Riemer, \& Toon, 2001). Nesse aspecto, existem diferenças importantes entre os estudos. Riemer e Toon (2001) e Çakioglu (2003) analisaram atletas universitários (> 18 anos) de apenas uma modalidade esportiva (tênis e futebol, respectivamente). Já o presente estudo envolveu atletas de oito modalidades, com idades entre $15 \mathrm{e}$ 17 anos. Além disso, enquanto a amostra do presente estudo foi composta por atletas brasileiros de ambos os sexos, Çakioglu (2003) analisou atletas turcos do sexo masculino e Riemer e Toon (2001) analisou atletas americanos de ambos os sexos.

Andrew (2009) também testou a hipótese da congruência por meio da regressão linear múltipla com a interação entre os termos da liderança. Foram analisados atletas americanos de tênis com idades entre 18 e 24 anos ( $M=20,01 \pm 1,38$ anos). Os atletas responderam o QSA-L (idêntico ao presente estudo) e uma versão modificada da escala de liderança, elaborada por Zhang, Jensen e Mann (1997). Esse instrumento é composto por 60 itens distribuídos em seis dimensões, ao contrário da ELE que possui 40 itens e cinco dimensões. Diferentemente do presente estudo, Andrew (2009) encontrou que a congruência em apenas duas dimensões da liderança (treino e instrução e comportamento autocrático) causou impacto significativo na satisfação dos atletas. Dessa forma, esse pesquisador concluiu que a hipótese da congruência foi parcialmente confirmada.

O único estudo encontrado com atletas brasileiros foi Lopes (2006). Essa pesquisadora analisou atletas profissionais participantes da Superliga Feminina de Voleibol, utilizando o QSA-L e a versão modificada da escala de liderança, proposta por Zhang et al. (1997). Assim como nos estudos de Riemer e Toon (2001) e Çakioglu (2003), as análises de regressão múltipla realizadas por Lopes (2006) não demonstraram mudança significativa no $\mathrm{R}^{2}$ com a inserção da variável interação. Ou seja, a congruência entre a liderança preferida e percebida não modificou a satisfação dos atletas. Portanto, a hipótese da congruência foi rejeitada para todas as dimensões da satisfação. Mais uma vez, as diferenças entre o presente estudo e Lopes (2006) podem justificar a divergência nos resultados. Embora ambos tenhamos sido realizados com atletas brasileiros, Lopes (2006) avaliou apenas atletas do sexo feminino na modalidade voleibol. Além disso, esta pesquisadora investigou o esporte profissional e utilizou um instrumento para liderança diferente do presente estudo.

Embora o presente estudo tenha rejeitado a hipótese da congruência para uma das quatro dimensões da satisfação, este foi o trabalho que confirmou em maior amplitude a principal proposição do MML. Todas as cinco dimensões da liderança (TI, FP, SS, CA e CD) influenciaram a satisfação dos atletas, constituindo um achado inédito na literatura. Também se diferencia nesta pesquisa o número de modalidades investigadas. 
Enquanto a maior parte dos estudos investigou apenas uma modalidade, o presente estudo analisou atletas de oito modalidades esportivas (quatro coletivas e quatro individuais). Portanto, para o contexto avaliado, foi demonstrado que a satisfação dos atletas é dependente da congruência entre a liderança preferida e percebida, em três das quatro dimensões da satisfação, confirmando parcialmente a hipótese da congruência.

\section{Considerações Finais}

A liderança é um fenômeno social complexo derivado das relações entre as pessoas e depende da interação dos fatores pessoais do líder, dos liderados e do contexto. Portanto, trata-se de um fenômeno interacional em que os comportamentos são aprendidos a partir da relação entre os membros do grupo e o ambiente. Nesse sentido, Riemer e Toon (2001) sugeriram que a validade da hipótese da congruência seria uma função das condições situacionais. Dessa forma, a hipótese da congruência poderia não ser verdadeira em todas as situações ou contextos, mas seria dependente da interação dos fatores antecedentes da liderança. As pesquisas disponíveis na literatura parecem confirmar essa sugestão.

Nesse sentido, cabe destacar que o esporte escolar aqui investigado é caracterizado pela alta competitividade das equipes e por eventos organizados conforme as regras oficiais. Embora os atletas não sejam profis-

\section{Referências}

Andrew, D. (2004). The effect of congruence of leadership behaviors on motivation, commitment, and satisfaction of college tennis players (doctoral dissertation). Florida State University, USA.

Andrew, D. (2009). The impact of leadership behavior on satisfaction of college tennis players: a test of the leadership behavior congruency hypothesis of the multidimensional model of leadership. Journal of Sport Behavior, 32(3), 261-277. Recuperado de https://www.highbeam.com/doc/1G1-206532585.html

Aoyagi, M. W., Cox, R. H., \& McGuire, R. T. (2008). Organizational citizenship behavior in sport: relationships with leadership, team cohesion, and athlete satisfaction. Journal of Applied Sport Psychology, 20(1), 25-41. doi:10.1080/10413200701784858 sionais, eles buscam o alto rendimento esportivo. No presente estudo, os atletas treinavam, em média, quatro dias por semana com duração de mais de duas horas cada treino. $\mathrm{O}$ tempo médio de experiência em competições esportivas foi de 4,5 anos, ou seja, eles começaram a competir com aproximadamente 11 anos de idade.

Treinadores envolvidos no esporte escolar assumem a responsabilidade de desenvolverem as capacidades esportivas de seus atletas, conciliando a busca pelo melhor desempenho com o bem-estar físico, psicológico e social. Os resultados do presente estudo confirmaram que o comportamento dos treinadores tem um impacto relevante nas respostas psicológicas dos atletas (satisfação). Ao reconhecer os efeitos da liderança do treinador na satisfação dos atletas, estratégias podem ser adotadas a fim de potencializar os resultados positivos da sua experiência esportiva.

Estudos futuros poderão verificar os efeitos de variáveis pessoais e do ambiente na hipótese da congruência (por exemplo, sexo dos atletas e treinadores, nível de experiência e tipo de modalidade) e avançar nas discussões. Além disso, considerando que as relações interpessoais entre treinadores e atletas podem ter impacto significativo em ambos, sugere-se que os estudos de liderança incluam a análise da satisfação dos treinadores.

Barrow, J. (1977). The variables of leadership: a review and conceptual framework. Academy of Management Review, 2(2),231-251.doi:10.5465/AMR.1977.4409046

Bass, B. M. (2008). The bass handbook of leadership: theory, research, and managerial applications. New York, NY: Simon \& Schuster.

Borrego, C. M. C., Leitão, J. C., Alves, J., Silva, C., \& Palmi, J. (2010). Análise confirmatória do Questionário de Satisfação do Atleta: versão Portuguesa. Psicologia: Reflexão e Crítica, 23(1), 110-120. doi:10.1590/S0102-79722010000100014

Çakioglu, A (2003). Leadership and satisfaction in soccer: examination of congruence and players' position (master's thesis). Middle East Technical University, Turkey. Chelladurai, P. (1978). A contingency model of leadership in athletics (doctoral dissertation). University of Waterloo, Canada. 
Chelladurai, P. (1984). Discrepancy between preferences and perceptions of leadership behavior and satisfaction of athletes in varying sports. Journal of Sport Psychology, 6(1), 27-41.

Chelladurai, P. (1993). Leadership. In R. N. Singer, M. Murphey, \& L. K. Tennant (Eds.), Handbook of research on sportpsychology (pp. 647-671). NewYork, NY: Macmillan.

Chelladurai, P. (2012). Leadership and manifestations of sport. In S. M. Murphy (Ed.), The Oxford handbook of sport and performance psychology (pp. 328-342). New York, NY: Oxford University Press.

Chelladurai, P. (2007). Leadership in sports. In G. Tenenbaum, \& R. C. Eklund (Eds.), Handbook of sport psychology (pp. 113-135). Hoboken, NJ: John Wiley \& Sons.

Chelladurai, P., \& Carron, A. V. (1983). Athletic maturity and preferred leadership. Journal of Sport Psychology, 5(4), 371-380. Recuperado de http://journals.humankinetics.com/jsep-back-issues/JSEPVolume5Issue4December/AthleticMaturityandPreferredLeadership

Chelladurai, P., \& Riemer, H. (1997). A classification of facets of athlete satisfaction. Journal of Sport Management, 11, 133-159.

Chelladurai, P., \& Riemer, H. (1998). Measurement of leadership in sports. In J. L. Duda (Ed.), Advances in sport and exercise psychology measurement (pp. 227-253). Morgantown, WV: Fitness Information Technology.

Chelladurai, P., \& Saleh, S. D. (1980). Dimensions of leader behavior in sports: development of a leadership scale. Journal of Sport Psychology, 2(1), 34-45.

Chelladurai, P., \& Saleh, S. D. (1978). Preferred leadership in sports. Canadian Journal Applied Sport Sciences, 3, 85-92.

Comitê Olímpico Brasileiro - COB. (2013) Acreditando nos nossos jovens... Recuperado de http://jogosescolares.cob.org.br/o-evento

Danielson, R. R., Zelhart Jr, P. F., \& Drake, C. J. (1975). Multidimensional scaling and factor analysis of coaching behavior as perceived by high school hockey players. Research Quarterly, 46(3), 323-334.

Day, D. V., \& Antonakis, J. (2012). Leadership: past, present and future. In D. V. Day, \& J. Antonakis (Eds.), The nature of leadership (pp. 3-25). Thousand Oaks, CA: SAGE Publications.

Halpin, A.W., \&Winer,B. J. (1957). A factorial study of the leader behavior description. In R. M. Stogdill, \&A. E. Coons (Eds.), Leader behavior: its description and measurement (pp. 39-51). Columbus, OH: The Ohio State University.
Hernandez, J.A.E., \&Voser, R. C. (2012).Validação da escaladeliderançapara oesporte:versãopreferência dos atletas. Psicologia: Ciência e Profissão, 32(1), 142-157. doi:10.1590/S1414-98932012000100011

Horn, T. S., Bloom, P., Berglund, K. M., \& Packard, S. (2011). Relationship between collegiate athletes' psychological characteristics and their preferences for different types of coaching behavior. The Sport Psychologist, 25, 190-211.

Horne, T., \& Carron, A. V. (1985). Compatibility in coach-athlete relationships. Journal of Sport Psychology, 7(2), 137-149. Recuperado de http:// journals.humankinetics.com/jsep-back-issues/ JSEPVolume7Issue2June/CompatibilityinCoachAthleteRelationships

Jowet, S. (2003). When the "honeymoon" is over: a case study of a coach-athlete dyad in crisis. The Sport Psychologist, 17(4), 444-460.

Lopes, M. C. (2006). A relação do perfil de liderança dos treinadores de voleibol com a satisfação das atletas na Superliga Feminina 2004/2005 (dissertação de mestrado). Escola de Educação Física, Fisioterapia e Terapia Ocupacional, Universidade Federal de Minas Gerais, Belo Horizonte.

Lopes, M. C., Samulski, D. M., \& Silva, L. A. (2007). Validação do questionário de satisfação do atleta: versão liderança. Revista Brasileira de Ciência e Movimento, 15(4), 47-56.

Olympiou, A., Jowett, S., \& Duda, J. 1. (2008). The psychological interface between the coach-created motivational climate and the coach-athlete relationship in team sports. The Sport Psychologist, 22(4), 423-438. Recuperado de https://www. researchgate.net/publication/229096790_The_Psychological_Interface_Between_the_Coach-Created_Motivational_Climate_and_the_Coach-Athlete_Relationship_in_Team_Sports

Parry, K. W., \& Bryman, A. (2006). Leadership in organizations. In S. R. Clegg, C. Hardy, T. B. Lawrence, \& W. R. Nord (Eds.), The SAGE handbook of organizational studies (pp. 447-468). London: SAGE.

Riemer, H. A., \& Chelladurai, P. (1998). Development of the Athlete Satisfaction Questionnaire (ASQ). Journal of Sport and Exercise Psychology, 20(2), 127-156. Recuperado de http://journals.humankinetics.com/ jsep-back-issues/JSEPVolume20Issue2June/DevelopmentoftheAthleteSatisfactionQuestionnaireASQ 
Riemer, H. A., \& Chelladurai, P. (1995). Leadership and satisfaction in athletics. Journal of Sport and Exercise Psychology, 17(3), 276-293. Recuperado de http:// journals.humankinetics.com/jsep-back-issues/ JSEPVolume17Issue3September/LeadershipandSatisfactioninAthletics

Riemer, H. A., \& Toon, K. (2001). Leadership and satisfaction in tennis: examination of congruence, gender and ability. Research Quarterly for Exercise and Sport, 72(3), 243-256. doi:10.1080/02701367.2001.10608957

Rodrigues, V. M. (2014). Liderança do treinador e satisfação de atletas escolares (tese de doutorado). Centro de Ciências Humanas e Letras, Universidade Federal da Paraíba, Paraíba.

Thompson, I. (2003). Jogos Estudantis Brasileiros JEBs. In P. C. Lamartine (Org.), Atlas do esporte no Brasil: atlas do esporte, educação física e atividades físicas de saúde e lazer no Brasil (pp. 807-809). Rio de Janeiro, RJ: Shape.

Zhang, J., Jensen, B. E., \& Mann, B. L. (1997). Modification and revision of the leadership scale for sport. Journal of Sport Behavior, 20(1), 105-121.

Vinicius de Matos Rodrigues

Doutor. Docente do Instituto Federal da Bahia, Porto Seguro - BA. Brasil.

E-mail: vinicius.ifba@gmail.com

\section{Ana Alayde Werba Saldanha}

Doutora. Docente da Universidade Federal da Paraíba, João Pessoa - PB. Brasil.

E-mail: analayde@gmail.com
Endereço para envio de correspondência: Instituto Federal da Bahia, Campus Porto Seguro, BR 367, Km 57,5. Fontana I. CEP: 45815-000. Porto Seguro - BA. Brasil.

Recebido 31/10/2014

Reformulação 13/11/2015

Aprovado 19/07/2016

Received $10 / 31 / 2014$

Reformulated $11 / 13 / 2015$

Approved 07/19/2016

Recibido 31/10/2014

Reformulado 13/11/2015

Aceptado 19/07/2016

Como citar: Rodrigues, V. M., \& Saldanha, A. A. W. (2016). Liderança e satisfação no esporte escolar: teste da hipótese da congruência do Modelo Multidimensional de Liderança. Psicologia: Ciência e Profissão, 36(3): 653-667. doi:10.1590/1982-3703001492014

How to cite: Rodrigues, V. M., \& Saldanha, A. A. W. (2016). Leadership and satisfaction in young athletes: testing the hypothesis of congruence of the Multidimensional Model of Leadership. Psicologia:Ciênciae Profissão, 36(3):653-667. doi:10.1590/1982-3703001492014

Cómo citar: Rodrigues, V. M., \& Saldanha, A. A. W. (2016). El liderazgo y la satisfacción en el deporte escolar: probando la hipótesis de la congruencia del Modelo Multidimensional de Liderazgo. Psicologia: Ciênciae Profissão, 36(3): 653-667. doi:10.1590/1982-3703001492014 